\title{
Statistical Model for the Classification of the Wavelet Transforms of T-ray Pulses
}

\author{
X.X. Yin, B.W.-H. Ng, B. Ferguson, S.P. Mickan and D. Abbott \\ Center for Biomedical Engineering, The University of Adelaide, SA 5005, Australia \\ xxyin@eleceng.adelaide.edu.au
}

\begin{abstract}
This study applies Auto Regressive (AR) and Auto Regressive Moving Average (ARMA) modeling to wavelet decomposed terahertz pulsed signals to assist biomedical diagnosis and mail/packaging inspection. T-ray classification systems supply a wealth of information about test samples to make possible the discrimination of heterogeneous layers within an object. In this paper, the classification of normal human bone (NHB) osteoblasts against human osteosarcoma (HOS) cells and the identification of seven different powder samples are demonstrated. A correlation method and an improved Prony's method are investigated in the calculation of the AR and ARMA model parameters. These parameters are obtained for models from second to eighth orders and are subsequently used as feature vectors for classification. For pre-processing, wavelet de-noising methods including the SURE (Stein's Unbiased Estimate of Risk) and heuristic SURE soft threshold shrinkage algorithms are employed to de-noise the normalised T-ray pulsed signals. A Mahalanobis distance classifier is used to perform the final classification. The error prediction covariance of $A R / A R M A$ modeling and the classification accuracy are calculated and used as metrics for comparison.
\end{abstract}

\section{T-ray imaging and inspection sys- tems}

Terahertz radiation, also called T-rays, is a collective term to describe the part of the electromagnetic spectrum from $0.1 \mathrm{THz}$ to $10 \mathrm{THz}$. Compared to X-rays, T-rays are non-invasive and non-ionizing. The vibrational spectra characteristics in this frequency range make it a promising modality for clinical diagnosis. With the rapid improvement of T-ray detectors and sources, T-ray technology makes it possible to image opaque objects including the inspection of tumor cells from normal tissues [3]. However, signal processing is a relatively unexplored technique for T-ray systems, despite much effort having been devoted to system improvement [3]. This includes progress towards wavelet de-noising of optical terahertz pulse imaging data and classification of T-ray signals [6].

The wavelet transform is a popular technique suited to the analysis of very brief signals, especially signals with sudden and unpredictable changes that often carry the most interesting information [1]. The major foreseeable advantage of a wavelet-based approach is the superior time-frequency localization characteristics that are well-matched to the requirements for the short-duration T-ray pulse signals.

This paper adds to the important T-ray and wavelet application research fields by applying linear parameter modeling to wavelet decomposition of T-ray signals for optimum feature extraction with a goal of classifying biomedical samples and package inspection data.

\section{Approach}

\subsection{Wavelet de-noising of T-ray pulsed re- sponses}

Wavelet de-noising is critical in the pre-processing of T-ray signals in order to obtain accurate classification. Wavelet shrinkage de-noising has been well-studied, and they have been shown to be statistically optimal [1]. The basic steps in wavelet shrinkage are to calculate the discrete wavelet packet transform (DWPT) or discrete wavelet transform (DWT) of a signal, perform a thresholding operation on the detail coefficients and then apply the corresponding inverse transform (IDWPT or IDWT) to reconstruct the de-noised signal.

In this paper, the SURE method and the 'heurstic' SURE method are used separately to estimate the soft threshold $\lambda^{S}$ [1] for the cancerous and powder 
classification experiments, respectively. In evaluating the amount of background noise removed by wavelet shrinkage de-noising, the signal-to-noise ratio, SNR, is used. The SNR in these experiments is calculated from the following equation:

$$
\operatorname{SNR}(\mathrm{dB})=10 \log _{10} \frac{\sum_{k=0}^{N-1} r(k)^{2}}{\sum_{k=0}^{N-1}[r(k)-d(k)]^{2}}
$$

where $r(k)$ are the raw T-ray signal samples and $d(k)$ are the de-noised signal samples.

In this paper, the SNR for one pixel of a soda powder sample at a thickness of $3 \mathrm{~mm}$ and for one pixel of cancerous sample after de-noising are calculated to be $17 \mathrm{~dB}$ and $21 \mathrm{~dB}$, respectively. The two results indicate that a significant amount of noise has been removed from the T-ray signals, which otherwise can degrade the classification performance.

\subsection{AR model parameter estimation}

The forward linear prediction auto-regressive (AR) model is given by a linear difference equation in the time domain:

$$
\hat{x}[n]=-\sum_{i=1}^{P} a_{i} x[n-i]+w[n]
$$

where $\hat{x}[n]$ represents the current prediction, which is predicted by previous $P$ observations of the sequence. The variables $a_{i}$ are the coefficients of AR model. The prediction filter is driven by an uncorrelated white noise process, $w[n]$.

The autocorrelation method estimates a required correlation matrix from a data sequence of length $N_{s}$ [5]. The least square form of the Yule-Walker equation is used to estimate the parameters of the AR model:

$$
\left(\mathbf{X}^{* T} \mathbf{X}\right) \mathbf{a}=\left[\mathcal{S}_{A} \mathbf{0}\right]^{T}
$$

where $\mathbf{a}=\left[a_{0} \ldots a_{P}\right]^{T}$ and $\mathcal{S}$ is the minimum sum of squared errors. The data matrix $\mathbf{X}$ for the autocorrelation method is determined by the selection the end points as $n_{I}=0$ and $n_{F}=N_{s}+P-1$.

The prediction error variance is calculated as Eq. 4.

$$
\sigma_{\epsilon P}^{2}=\frac{1}{n_{F}-n_{I}+1} \mathcal{S}
$$

\subsection{ARMA model parameter estimation}

ARMA modeling is a combination of AR and moving average (MA) models. The MA model is developed from the following difference equation:

$$
x[n]=\sum_{i=0}^{Q} b_{i} w[n-i]
$$

Here, output $x[n]$ is seen as a sum of weighted average of the past $Q$ input samples of a white noise process $w(n)$. The variables $b_{i}$ are the coefficients of MA model. The full ARMA model equation can be obtained by combining by both AR and MA models, as below:

$$
\sum_{i=0}^{P} a_{i} x[n-i]=\sum_{j=0}^{Q} b_{j} w[n-j], \quad a_{0}=1 .
$$

The estimation of the AR and MA model parameters can be derived from the least squares method mentioned above. The estimation of the AR part of the ARMA with the autocorrelation method has already been described above; the MA parameters can be estimated by Durbin's method [5]. This method is based on the basic Prony's procedure [5]: assume that $\check{x}[n]=$ $x[n]$ if $n=0,1, \ldots N_{s}-1, N_{s}=P+Q+1$ with $P=Q$. For most situations, $N_{s}>P+Q+1$, then the YuleWalker matrix equation of the ARMA modeling can be partitioned as in Eq. (7):

$$
\left(\mathbf{X}_{B} \mathbf{X}_{A}\right)^{T} \mathbf{a}=\left[\mathbf{b} \mathbf{e}_{A}\right]^{T}
$$

Here, $\mathbf{a}=\left[a_{0} \ldots a_{P}\right]^{T}, \mathbf{b}=\left[b_{0} \ldots b_{Q}\right]^{T}, P=Q ; \mathbf{e}_{A}$ is the prediction error that is defined as $\mathbf{e}_{A}=x[n]-\check{x}[n]$.

An improvement of basic Prony's method is Durbin's method. The related AR coefficient vector $\mathbf{a}$ is calculated to minimise $\mathbf{S}_{A}=\left|\mathbf{e}_{A}\right|^{2}$, where $\mathbf{e}_{A}=\left[\mathbf{X}_{A}\right] \mathbf{a}$ from Eq. (7). The Yule-Walker equations for the AR model is then $\left(\mathbf{X}_{A}^{* T} \mathbf{X}_{A}\right) \mathbf{a}=\left[\mathcal{S}_{A} 0\right]^{T}$. The difference lies in the calculation of MA parameters $\left(b_{1}, b_{2}, \cdots b_{k}\right)$, which considers the existence of error term in Eq. (7) $\mathbf{X}_{A} \mathbf{a}=\mathbf{b}$, instead of forcing $\mathbf{b}$ to match the left side of the equation. Detailed derivations can be found in [5]. The calculation of the coefficient vector $\mathbf{b}$ is carried out in two stages:

1. The order of AR model, $P$, is selected to be five times the MA model order $Q$. The coefficient vector $\mathbf{a}$ is obtained from the solution of the YuleWalker equation normalized by the gain, $\sqrt{a_{0}}$, i.e. substitute $\mathcal{S}$ with unity in Eq. (3).

2. Use the coefficient vector a, derived from the first stage, as the data to construct the data matrix for a MA model order $Q$. The autocorrelation method guarantees the stability of computations.

\subsection{Feature extraction}

Feature extraction is an important step in all but the simplest classification problems. The objective of feature extraction is to isolate the critical features from the T-ray signals to facilitate good classification performance. 
In the current approach, the feature extraction process involves several steps. First, the signals are pre-processed by wavelet shrinkage denoising. Then, DWPT or DWT is performed, using the biorthogonal biorSpline wavelet of order 6.8 and orthogonal Daubechies wavelet of order 8 , respectively. The transforms are repeated for depths from 2 to 4 . Subsequently, the autocorrelation and Durbin's methods are used to calculate the parameters for AR and ARMA models, respectively. These calculations are repeated for two different AR and ARMA model orders. Finally, the average of the model coefficients for the two different orders of AR or ARMA models are computed for each of the three different levels of wavelet transform. The model parameter averages are then joined to produce feature vectors with a dimension of 2,3 or 4, depending on the depth of wavelet transform chosen.

The motivation of using AR/ARMA models is to reduce the final dimensionality of the feature vectors. With these models, it is possible to compress the information in the wavelet coefficients into a few model parameters. Owing to the dimensional variety of AR/ARMA parameters corresponding to the various AR/ARMA orders, the average of AR/ARMA parameters is used to further consolidate the varying dimensions of AR/ARMA parameters into a fixed dimension for the final feature vectors. The overall dimensionality of the feature vectors depend only on the depth of the wavelet transform chosen.

\subsection{Classification}

The classification is realized by a Mahalanobis distance classifier [4]. The Mahalanobis distance is defined as the distance from the mean value of the pointed class to a given point, where the given class is normalized by the different training vectors along their respective directions [4]. For a given class, $i$, the distance from a point $x$ from the class mean $\mu_{i}$, is defined as

$$
d_{i}(x)=\sqrt{\left(x-u_{i}\right)^{T} \sum_{i}^{-1}\left(x-u_{i}\right)} .
$$

The minimum Mahalanobis distance is then calculated to select the class of samples and get final classification.

\section{Results and discussion}

To verify the performance of the proposed approach, it is applied to two different sets of T-ray data. One set of data contains 50 responses from samples corresponding to each class: normal human bone (NHB) osteoblasts, human osteosarcoma (HOS) cells, and a empty holder. The other set of data contains 50 responses from samples corresponding to each powder class: granulated sugar, powdered sugar, salt, talcum, sand, baking soda, flour, and the empty holder. Different wavelet families (biorthogonal and orthogonal) were chosen for the two sets of data in the experiments.

In our feature extraction scheme, the depth of wavelet transform is a central parameter. In the course of our experiments, it has been found that a depth of 3 generally gives the best classification performance. For the sake of brevity, only the results based on this choice of wavelet transform depth are presented in this paper.

\subsection{Classification of NHB and HOS cells}

Normal human bone (NHB) cells were obtained from patients and cultured from small pieces of trabecular bone for 4-6 weeks to obtain a confluent culture. Human osteosarcoma (HOS) cells were cultured from an immortalized cell line and it took 1 week to obtain a confluent culture. Here, $25 \mathrm{ml}$ polystyrene flasks are used as holders to culture the cells under a $5 \%$ carbon dioxide environment and a temperature of $37^{\circ} \mathrm{C}$. To obtain T-ray image data, the flasks were placed on a $\mathrm{x}-\mathrm{y}$ rotation stage allowing the cell media solution to run off. A THz image was obtained at 10 different positions to provide spectroscopic data, with a distance interval of $50 \mu \mathrm{m}$. The above procedure was performed for each of the three flasks - normal cells, cancerous cells and empty holders - and iterated a further 5 times until 50 pixels of the $\mathrm{THz}$ response were obtained for all three flasks [2]. It should be emphasized that the rationale for choosing bone cancer cells for this research is because techniques for culturing bone cell growth on a Petri dish are well-established and readily accessible. Our positive results with T-ray detection of cancerous bone cells will motivate future research to explore other classes of cancer cells. While the amount of data obtainable was limited by practical experiment apparatus, the set of available data should be sufficient for our investigative purposes.

The averages of two different orders of AR coefficients from a depth 3 wavelet decomposition are uses as features. The AR parameters are estimated from all the wavelet subbands (coarse and details). The Mahalanobis distance classifier is trained using half of the T-ray responses (25 pixel responses) corresponding to each of these classes - cancer cells, normal cells and the empty control flask - then the remaining half of the pixel responses were classified. Error prediction covariance and accuracy of classification at different orders of AR modeling and the different levels of wavelet transform are calculated and employed as metrics for 
comparison.

As stated above, different combinations of AR orders at a wavelet transform depth of 3 in better classification performance than other depths. Of all the combinations of the AR orders considered $\left(2^{\text {nd }}\right.$ to $\left.8^{\text {th }}\right)$, the $3^{\text {rd }}$ and the $5^{\text {th }}$ order AR models produced the best classification performance with a classification accuracy $93 \%$ and the minimum error covariance $4 \times 10^{-19}$, which is extracted by comparison with all the different combination of $\mathrm{AR}$ orders.

\subsection{Classification of powder samples}

In this experiment, the responses are obtained from samples of different thicknesses: $2 \mathrm{~mm}, 3 \mathrm{~mm}$ and 4 $\mathrm{mm}$. The data from seven classes of powders at thicknesses of $2 \mathrm{~mm}$ and $4 \mathrm{~mm}$ are used to train the classifier, and the data of powder samples at a thickness of $3 \mathrm{~mm}$ are used to test the classifier.

It has been found that features obtained from the ARMA model separated the different classes quite effectively. The accuracy of the classification can be improved by at least $3 \%$ over either AR or MA models alone. The squared error variance we obtained is at most $3 \times 10^{-14}$, which is minimum among the various methods for calculating AR or ARMA parameters with the same conditions, including: correlation methods, covariance methods, modified covariance methods and the basic Prony's method. It was found that the combination of the $2^{\text {nd }}$ and $4^{\text {th }}$ orders of ARMA models of wavelet decomposed T-ray signals obtained the best classification accuracy. The combination of the two modeling coefficients at these two orders achieved a classification accuracy of $98 \%$.

\section{Conclusion}

In this paper, an approach yielding good classification performance is presented. By using the same DWPT- or DWT-based techniques, it has been found that AR/ARMA models of wavelet coefficients from T-ray pulsed responses is capable of achieving classification accuracies of $93 \%$ for cancerous and normal cell samples and 98\% for powder samples. The use of Durbin's algorithm in estimating the ARMA coefficients also yielded error variances at least as low as other methods in the powder experiment. Despite the improved classification performance, the approach presented requires much lower computation costs when compared to FFT-based techniques, which usually have to be coupled with other computationally expensive algorithms to achieve their optimum performance.
The ultimate aim of the current research is to achieve heterogenous layer classification in a volume space based on T-ray imaging. Work is ongoing to transfer these results to scenarios where in vivo detection will require discrimination through a number of heterogeneous layers. A 3D tomographic classification for the homogeneous layers have been achieved. The paper [7] reports on more results from work on this front.

In conclusion, T-ray pulsed signals are capable of being effectively exploited by applying computation models in the wavelet transform domain.

\section{References}

[1] D. L. Donoho. De-noising by soft thresholding. IEEE Transactions on Information Theory, 41(3):613-627, 1995

[2] B. Ferguson, H. Liu, S. Hay, D. Findlay, X.-C. Zhang, and D. Abbott. In vitro osteosacoma biosensing using $\mathrm{THz}$ time domain spectroscopy. Proc. of SPIE BioMEMS and Nanotechnology, 5275:304-316, 2004.

[3] T. Löffler, K. Siebert, S. Czasch, and H.G. Bauer, T.and Roskos. Visualization and classification in biomedical terahertz pulsed imaging. Physics in Medicine and Biology, 47(2002):3847-3852, 2002.

[4] J. Schürmann. Pattern Classification: A Unified View of Statistical and Neural Approaches. John Wiley and Sons, Inc., New York, 1996.

[5] C.W. Therrien and A.V. Oppenheim. Discrete Random Signals and Statistical Signal Processing. Prentice Hall, New Jersey, USA, 1992.

[6] X.X. Yin, B. W.-H. Ng, B. Ferguson, S. P. Mickan, and D. Abbott. One dimensional wavelet transforms and their application to T-ray pulsed signal identification. In Proc. of SPIE Photonics: Design, Technology, and Packaging, volume 6038, pages 499-509, Brisbane, Australia, 2006.

[7] X.X. Yin, B. W.-H. Ng, B. Ferguson, S. P. Mickan, and D. Abbott. Wavelet based segment detection and feature extraction for 3D T-ray CT pattern classification. In IEEE Signal Processing Society: 12th Digital Signal Processing Workshop 4th signal Processing Education Workshop, Wyoming, USA, 2006, (submitted). 